\title{
The Density Determination of Small Solid Objects by a Simple Float Method-II
}

\author{
R. S. Davis* \\ National Bureau of Standards, Washington, DC 20234
}

January 18, 1982

\begin{abstract}
The measurement of density changes in solid samples of less than one gram is often of practical interest. We describe here such relative measurements having a precision of a few parts in $10^{4}$ using a newly reported apparatus. Comparisons of results with theory are presented.
\end{abstract}

Key words: density; density changes; density of solids; small samples.

\section{Introduction}

The preceding paper $[1]^{1}$ describes the construction and use of apparatus for determining the absolute density of small solid objects. It is often, however, of interest to find small differences in the relative densities among a set of samples. It is generally true that the density ratios of nearly equal samples can be found with higher accuracy than the density of any one sample can be measured absolutely. An example of the application of the apparatus of Schoonover [1] to this important class of measurement follows. The results make possible a qualitative comparison of the performance of this apparatus with a similar one developed by Franklin and Spal [2].

\section{The problem}

Four samples, $\mathrm{A}, \mathrm{B}, \mathrm{C}$, and $\mathrm{D}$, of crystalline $\mathrm{Al}_{2} \mathrm{O}_{3}$ (sapphire) were made available to us for evaluation. ${ }^{2}$ Sample $A$ was untreated and had a density of $3.986 \mathrm{~g}^{\bullet} \mathrm{cm}^{-3}$ based on x-ray measurements. Sample B, which was slightly pink in color, had $\mathrm{Ti}^{+3}$ substituted for $\mathrm{Al}^{+3}$ in about $0.1 \%$ of the aluminum sites of the pure material. To produce sample $\mathrm{C}$, a specimen similar to $\mathrm{B}$ had been heated at $1600^{\circ} \mathrm{C}$ for 1 day, which oxidized the dopant to the $\mathrm{Ti}^{+4}$ state causing the color to disappear. Finally, sample D was identical to $\mathrm{C}$ except that it was annealed in air for 190 hours at $1400{ }^{\circ} \mathrm{C}$.

\footnotetext{
*Center for Absolute Physical Quantities, National Measurement Laboratory

${ }^{1}$ Figures in brackets indicate literature references at the end of this paper.

${ }^{2}$ The author is indebted to Dr. Arthur Heuer of Case Western Reserve University for these samples, which were remnants of research carried out in his department.
}

This last sample is white, indicating the presence of $\mathrm{TiO}_{2}$ (rutile) precipitate.

Of particular interest was the density of sample $\mathrm{C}$ because this value was thought at the time of these measurements to depend strongly on the role of the charge concentration defects; i.e. whether aluminum vacancies are present (leading to a calculated density of

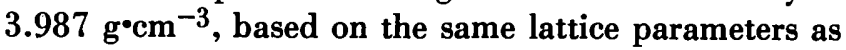
pure $\mathrm{Al}_{2} \mathrm{O}_{3}$ ) or whether interstitial oxygen is formed (raising the calculated density to $3.998 \mathrm{~g}^{\bullet} \mathrm{cm}^{-3}$ ). Other types of measurements by the group at Case Western Reserve strongly support the interstitial oxygen hypothesis [3]. ${ }^{3}$

\section{Experimental}

\subsection{Choice of Stem Diameter}

Since the expected change in the density of sample $\mathrm{C}$ relative to $A$ was thought to be $+30 \times 10^{-4}$, the experiment was designed to have a relative precision of at least $3 \times 10^{-4}$ in determining densities. The samples were adjusted by abrasion to have a nominal mass of $250 \mathrm{mg}$ and to be within $50 \mu \mathrm{g}$ of equality, after which they were weighed to determine their masses exact to $5 \mu$.

According to eq (8) of Ref. [1], the coated wire need only be $8.3 \times 10^{-3} \mathrm{~cm}$ in diameter $(0.003$ inch) which would give a theoretical precision of relative density of about $4 \times 10^{-6}$ but would certainly produce an enormous drift rate in the equilibrium position of the float due to temperature changes in the upper liquid. A wire of 0.51 $\mathrm{mm}$ diameter (0.020 inch) was therefore chosen as being

${ }^{3}$ This work was published subsequent to the measurements described here. 
the largest which would serve the experimental requirements.

\subsection{Measurements}

From equilibrium equations similar to (1) and (2) of Ref. [1], it is easy to show that the volume difference between two samples, e.g. $A$ and $B$, influences the equilibrium position of the float through the following relation:

$$
\Delta V \equiv V_{\mathrm{B}}-V_{\mathrm{A}}=\frac{W\left(\varrho_{H}-\varrho_{L}\right)}{\varrho_{L}}\left(\mathrm{O}_{\mathrm{B}}-\mathrm{O}_{\mathrm{A}}\right)+\frac{\mathrm{B}-\mathrm{A}}{\varrho_{L}}
$$

where $W$ is the cross-sectional area of the coated wire and the other terms have the same sense as in [1].

The ratio of densities is then given by

$$
\frac{\mathrm{e}_{\mathrm{B}}}{\mathrm{e}_{\mathrm{A}}}=\frac{\mathrm{B}}{\mathrm{A}\left(1+\Delta V \frac{\mathrm{e}_{\mathrm{A}}}{\mathrm{A}}\right)}
$$

Our hope was to determine $\Delta V$ through the relationships in eq (1) by using handbook values for $\varrho_{L}$ and $\varrho_{H}$, and micrometer measurements to determine $W$. The experience reported by Frankin and Spal was somewhat discouraging, however. The latter paper describes huge daily variations in the value of $W\left(\varrho_{H}-\varrho_{L}\right)$ inferred from float measurements; i.e., with a measured precision of $2 \times 10^{-4} \mathrm{~g} \cdot \mathrm{cm}^{-1}$ for a given run, values as large as 2.6 times and as small as 1.3 times greater than the theoretical value of $6.6 \times 10^{-4} \mathrm{~g}^{\circ} \mathrm{cm}^{-1}$ were found from experiment to experiment. [To circumvent this difficulty, they used a clever scheme involving the measurement of each unknown sample against six standards of equal density (near that of the unknown) and masses nominally equal but varying over a span of about 1.3 percent.]

In the work reported here samples $A, B, C$ and $D$ were placed on the float at regular intervals in a symmetric series and the equilibrium positions noted by viewing with a cathetometer as described in [1]. During the measurements, the equilibrium position of the float drifted upwards with time.

At the conclusion of the measurements, the equilibrium positions of each sample were plotted against time and the points fit with the least squares line. All four such lines had identical slopes within experimental uncertainty. At the same arbitrary instant near the midpoint of the measurements, the value of the equilibrium position was predicted from the four curves. It is worthwhile noting that the standard deviations of the predicted points were between $1.8 \times 10^{-3} \mathrm{~cm}$ and $3.0 \times 10^{-3} \mathrm{~cm}$ whereas the cathetometer itself had a repeatability of $0.5 \times 10^{-3} \mathrm{~cm}$. Thus for these measurements, the cathetometer does not limit the precision.

The pertinent data and results are shown in Table 1. The error bars represent a single standard deviation with no contribution being added for deviations of the buoyant forces from naive theoretical values.

The unexpected result for $e_{C} / \varrho_{A}$ prompted a check of the simple assumptions. To this end, three additional standards were fabricated-two of germanium and one of silicon. With the same stem described above but now using the three new standards to achieve an absolute determination of density as outlined in [1], the results shown in Table 2 were obtained.

\section{Discussion}

Subsequent to these measurements, data became available which led to an explanation of the unexpected results: The predictions of density for sample $C$ had assumed the lattice parameters of that crystal were the same as those of pure $\mathrm{Al}_{2} \mathrm{O}_{3}$. An actual measurement, however, revealed a significant lattice relaxation which, it is now believed, obscures the change in density to the extent that the revised predictions are presently within the precision of our measurements [3].

The close agreement between experimental and theoretical values for $\varrho_{L}$ and $W\left(\varrho_{H}-\varrho_{L}\right) / \varrho_{L}$ shown in table 2 is remarkable. The slight discrepancy in $\varrho_{L}$ values may reflect contamination of the upper liquid (methanol) with water, although this possibility was not followed up. The value obtained for $\varrho_{B} / \varrho_{A}$ is consistent in both sets of measurements justifying the simplifying assumptions made in obtaining results from the former set.

In short, none of the behavior which lead Franklin and Spal to suspect a density gradient in the upper liquid of their apparatus is apparent in the device used here. This result may reflect nothing more than a different choice of liquids but it is nevertheless worth noting. Beyond this, it is difficult to compare the performance of the apparatus used here with that reported in [2]. The latter is noteworthy for reporting a precision in ratio measurements equal to our own but on samples having one fifth the volume. It is not yet known whether the apparatus of Ref. [1] could do as well with smaller samples assuming the float volume and wire diameter were scaled down accordingly.

It is a pleasure to acknowledge the help, already alluded to, of Dr. Arthur Heuer of Case Western Reserve University in providing the samples used for this work, 


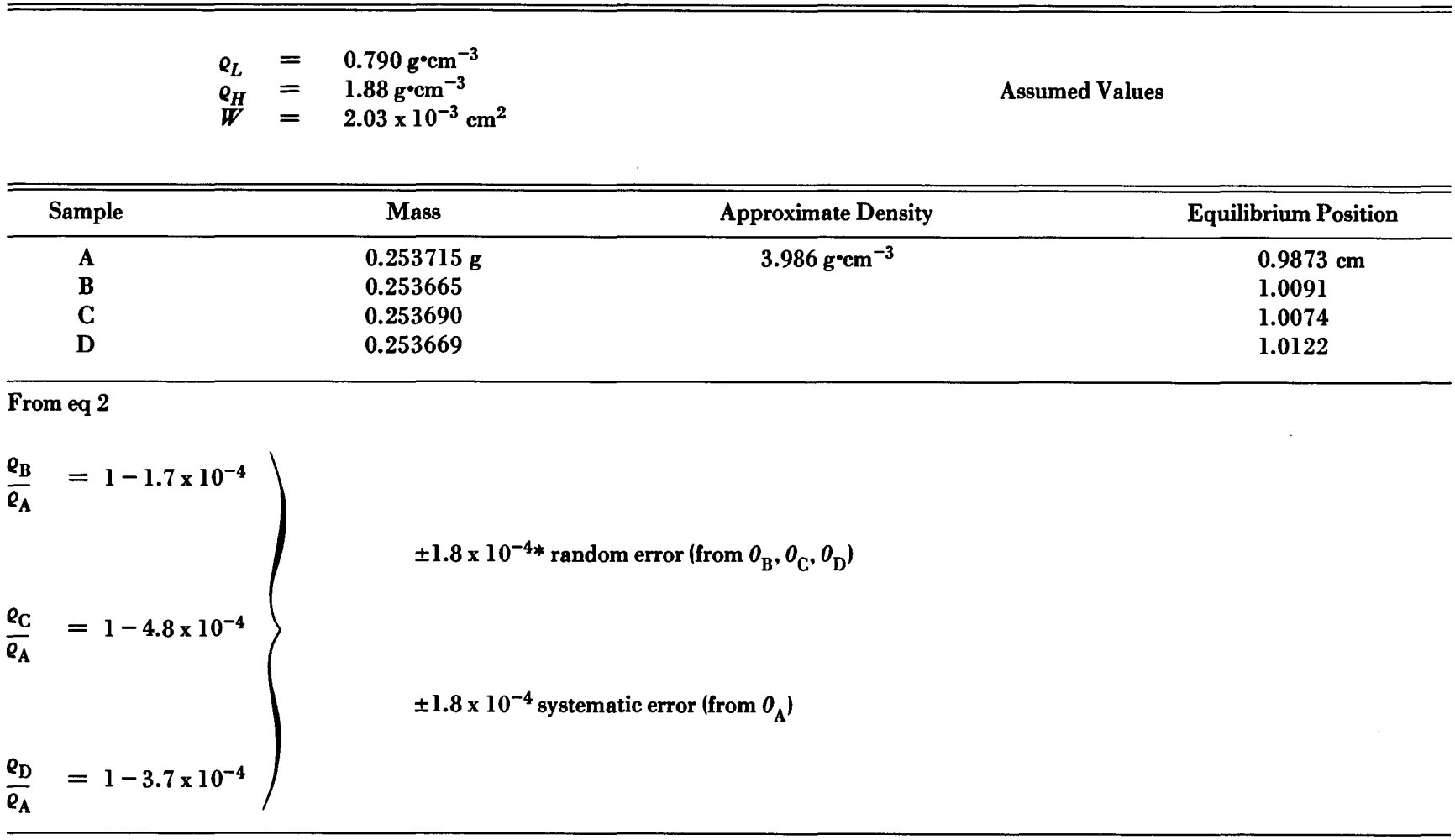

*errors are estimated at a level of one standard deviation.

in describing their history, and in patiently explaining the theoretical calculations of their density. I also wish to thank Randall Schoonover for advice in using the apparatus which he has developed.

\section{References}

[1] Schoonover, R.M. The density determination of small solid objects by a simple float method-I, J. Res. Nat. Bur. Stand. (U.S.) 87(3): ; 1982 May-June.

[2] Franklin, A. D.; Spal, R. A method for the precision comparison of the densities of small specimens. Rev. Sci. Instrum. 42 (12): 1827-1833; 1971 December.

[3] Phillips, D.S.; Mitchell, T. E.; Heuer, A. H. Precipitation in star sapphire III Chemical effects accompanying precipitation. Phil. Mag. A 42 (3): 417-432; 1980.
TABLE 2. Absolute density measurements of samples $A$ and $C$.

\begin{tabular}{ccl}
\hline \hline Standard & Mass & Density \\
\hline Ge 1 & $0.238260 \mathrm{~g}$ & $5.3270 \mathrm{~g}^{\circ} \mathrm{cm}^{-3}$ \\
Ge 2 & 0.234903 & 5.3270 \\
Si & 0.309219 & 2.3291 \\
& Temp $=22.20^{\circ} \mathrm{C}$ & \\
& & \\
\hline
\end{tabular}

Results:

$$
\text { Experimental }
$$

Assumed

$$
\begin{aligned}
& e_{\mathrm{A}}=3.9847 \pm 0.001 \mathrm{~g}^{\bullet} \mathrm{cm}^{-3 \mathrm{a}} \quad 3.986 \mathrm{~g}^{\bullet} \mathrm{cm}^{-3} \\
& \mathrm{e}_{\mathrm{C}}=3.9838 \pm 0.001 \mathrm{~g}^{\cdot} \mathrm{cm}^{-3} \\
& e_{L}=0.7897 \pm 0.0001 \mathrm{~g} \cdot \mathrm{cm}^{-3} \quad 0.7892 \mathrm{~g}^{\circ} \mathrm{cm}^{-3} \\
& \frac{W\left(e_{H}-e_{L}\right)}{e_{L}}=(2.83 \pm 0.02) \times 10^{-3} \mathrm{~cm}^{2} \quad(2.80 \pm 0.005) \times 10^{-3} \mathrm{~cm}^{2} \\
& \frac{Q_{C}}{Q_{A}}=1-2.3 \times 10^{-4} \pm 3.5 \times 10^{-4}
\end{aligned}
$$

aerrors are estimated at a level of one standard deviation. 